PROCEEDINGS OF THE

AMERICAN MATHEMATICAL SOCIETY

Volume 138, Number 3, March 2010, Pages 815-826

S 0002-9939(09)10186-7

Article electronically published on November 3, 2009

\title{
LAWS OF INERTIA IN HIGHER DEGREE BINARY FORMS
}

\author{
BRUCE REZNICK
}

(Communicated by Ken Ono)

\begin{abstract}
We consider representations of real forms of even degree as a linear combination of powers of real linear forms, counting the number of positive and negative coefficients. We show that the natural generalization of Sylvester's Law of Inertia holds for binary quartics but fails for binary sextics.
\end{abstract}

\section{INTRODUCTION AND OVERVIEW}

Let $F_{n, d}$ denote the set of real forms $p\left(x_{1}, \ldots, x_{n}\right)$ of degree $d, d \geq 1$. For even $d=2 s$, we consider representations of $p$ as a linear combination of the $2 s$-th powers of real linear forms:

$$
p\left(x_{1}, \ldots, x_{n}\right)=\sum_{j=1}^{r} \lambda_{j}\left(\alpha_{j 1} x_{1}+\cdots+\alpha_{j n} x_{n}\right)^{2 s}, \quad 0 \neq \lambda_{j} \in \mathbb{R} .
$$

If there are $a$ positive (and $b$ negative) coefficients among the $\lambda_{j}$ 's, then we say that (1.1) has badge $(a, b)$. Two linear forms are distinct if they (and their $2 s$-th powers) are not proportional; a representation is honest if its summands are pairwise distinct. Let $\mathcal{B}(p)$ denote the set of possible badges of honest representations of $p$. Badges are ordered componentwise: $(a, b) \preceq(c, d)$ if $a \leq c$ and $b \leq d$. Any minimal badge under this ordering is called a signature of $p$. The set of signatures of $p$ is denoted $\mathcal{S}(p)$; in case $p$ has a unique signature, we call it the signature of $p$ and denote it by $S(p)$. The length of $p$ is the minimum value of $a+b$ in $\mathcal{B}(p)$; it is the shortest possible representation (1.1). If $q$ is obtained from $p$ by an invertible linear change of variables, then $\mathcal{B}(q)=\mathcal{B}(p)$. Identities such as

$$
x^{2}+(x+y)^{2}+y^{2}=(x-y)^{2}+(x+2 y)^{2}-3 y^{2}=2\left(x+\frac{1}{2} y\right)^{2}+\frac{3}{2} y^{2}
$$

and

$$
(x+2 y)^{4}-4(x+y)^{4}+6 x^{4}-4(x-y)^{4}+(x-2 y)^{4}=24 y^{4}
$$

show that $\mathcal{B}(p)$ may have more than one element. We are also interested in sequences $\left\{p_{m}\right\} \subset F_{n, d}$ so that $p_{m} \rightarrow p$ coefficientwise and $S\left(p_{m}\right)=(a, b)$, but $(a, b) \notin \mathcal{S}(p)$, and we call this a jump signature sequence.

With the exception of a couple of remarks, we shall restrict our attention here to binary forms and rename the variables $(x, y)$. More generally, although the signs of the $\lambda_{j}$ 's are no longer relevant, the length may be defined over any field which

Received by the editors June 30, 2009.

2010 Mathematics Subject Classification. Primary 11E76, 15A21.

(C)2009 American Mathematical Society 
contains the coefficients of $p$, and for odd degree as well; see $[8$ for much more on this.

A simple consequence of Sylvester's Law of Inertia (Theorem 2.2) is that a quadratic form in $n$ variables has a unique signature. The main goal of this paper is to show that binary quartics have unique signatures, but a binary sextic might not.

The paper is organized as follows. In section two, we review a number of useful tools from the literature, many of them due to J. J. Sylvester. In section three, we prove some general results about signatures (Theorems 3.1 and 3.2). We find all possible signatures for $p \in F_{2,2 s}, s \geq 2$, though not all possible sets $\mathcal{S}(p)$. If $p \neq \pm \ell^{2 s}$ is a product of $2 s$ real linear forms, then it has a unique signature $(s, s)$. Associated to $p \in F_{2,2 s}$ is the catalecticant quadratic form $H_{p}$ in $s+1$ variables. If $(a, b) \in \mathcal{B}(p)$, then $S\left(H_{p}\right) \preceq(a, b)$. If $(a, b)$ and $(c, d)$ are both signatures of $p$, then $a+b+c+d \geq 2 s+4$. In section four, we show (Theorem 4.1) that binary quartics have unique signatures, and (Theorem 4.2) if $p$ has a definite quadratic factor, then $S(p)=S\left(H_{p}\right)$; otherwise, $S(p)=(2,2)$. We show that signatures for binary sextics are more complicated (Theorem 4.4): if $p(x, y)=x^{5} y+\alpha x^{3} y^{3}+x y^{5}$, $\alpha \in(-2,0]$, then $\mathcal{S}(p)=\{(2,3),(3,2)\}$. Section five contains some open questions and conjectures.

I thank Prof. Krishna Alladi and Prof. Manjul Bhargava and the participants in the Higher Degree Forms conference at the University of Florida in May 2009 for their encouragement and support of this work. I would also like to thank the referee for several valuable suggestions which improved the clarity of the exposition.

\section{TOOLS FROM THE LITERATURE}

Every form of degree $d$ is a linear combination of $d$-th powers of linear forms (see e.g. 6, p. 30]). A stronger result holds for binary forms.

Theorem 2.1. Any set $\left\{\left(\alpha_{j} x+\beta_{j} y\right)^{d}: 0 \leq j \leq d\right\}$ of pairwise distinct $d$-th powers over $\mathbb{R}$ is linearly independent and spans $F_{2, d}$.

Proof. The matrix of this set with respect to the basis $\left\{\left(\begin{array}{l}d \\ i\end{array}\right) x^{d-i} y^{i}: 0 \leq i \leq d\right\}$ is Vandermonde with determinant $\prod_{j<k}\left(\alpha_{j} \beta_{k}-\alpha_{k} \beta_{j}\right) \neq 0$.

In particular, $p \in F_{2,2 s}$ always has a representation $p(x, y)=\sum_{j=0}^{2 s} \lambda_{j}(x+j y)^{2 s}$, where $\lambda_{j} \in \mathbb{R}$. Thus $\mathcal{B}(p)$ always contains at least one badge $(a, b)$ with length $\leq 2 s+1$, and $\mathcal{S}(p)$ is non-empty.

Sylvester's Law of Inertia has several different expressions in the literature. This is the one which motivates this paper.

Theorem 2.2 (Sylvester's Law of Inertia). A real quadratic form in $n$ variables has a unique signature.

Proof. Suppose $p\left(x_{1}, \ldots, x_{n}\right)$ is a real quadratic form. Using the standard diagonalization, there is an invertible linear change of variables after which

$$
p\left(x_{1}, \ldots, x_{n}\right)=\sum_{j=1}^{a} x_{j}^{2}-\sum_{j=a+1}^{a+b} x_{j}^{2} .
$$

Suppose $p$ has another representation:

$$
p\left(x_{1}, \ldots, x_{n}\right)=\sum_{j=1}^{c+d} \lambda_{j}\left(\alpha_{j 1} x_{1}+\cdots+\alpha_{j n} x_{n}\right)^{2},
$$


where for simplicity, $\lambda_{j}>0$ for $1 \leq j \leq c$ and $\lambda_{j}<0$ for $c+1 \leq j \leq c+d$. We claim that $c \geq a$ and $d \geq b$. Indeed, if $c<a$, then there exists $\left(\bar{x}_{1}, \ldots, \bar{x}_{a}\right)$ so that

$$
\alpha_{j 1} \bar{x}_{1}+\cdots+\alpha_{j a} \bar{x}_{a}=0, \quad 1 \leq j \leq c .
$$

The two representations then imply a contradiction:

$$
0 \stackrel{\sqrt[(2.1)]{<}}{<} p\left(\bar{x}_{1}, \ldots, \bar{x}_{a}, 0, \ldots, 0\right) \stackrel{(2.2)}{\leq} 0 .
$$

A similar contradiction follows if $d<b$.

The diagonalization badge $(a, b)$ from (2.1) is the signature of the quadratic form $p$, both in the traditional sense and in our current definition. No such simple argument applies in higher degree, since diagonalization is usually not possible.

Sylvester proved the next theorem in the course of establishing the canonical expressions for binary forms. The original sources are [1, 9, 10. Modern discussions and proofs can be found in $[2,3,7,8$.

Theorem 2.3 (Sylvester). Suppose $p(x, y)=\sum_{j=0}^{d}\left(\begin{array}{l}d \\ j\end{array}\right) a_{j} x^{d-j} y^{j} \in \mathbb{C}[x, y]$ and

$$
h(x, y)=\sum_{j=0}^{r} c_{j} x^{r-j} y^{j}=\prod_{k=1}^{r}\left(\beta_{k} x-\alpha_{k} y\right), \quad r \leq d,
$$

is a product of pairwise distinct linear factors over $\mathbb{C}[x, y]$. Then there exist $\lambda_{k} \in \mathbb{C}$ so that

$$
p(x, y)=\sum_{k=1}^{r} \lambda_{k}\left(\alpha_{k} x+\beta_{k} y\right)^{d}
$$

if and only if

$$
\left(\begin{array}{cccc}
a_{0} & a_{1} & \cdots & a_{r} \\
a_{1} & a_{2} & \cdots & a_{r+1} \\
\vdots & \vdots & \ddots & \vdots \\
a_{d-r} & a_{d-r+1} & \cdots & a_{d}
\end{array}\right) \cdot\left(\begin{array}{c}
c_{0} \\
c_{1} \\
\vdots \\
c_{r}
\end{array}\right)=\left(\begin{array}{c}
0 \\
0 \\
\vdots \\
0
\end{array}\right) .
$$

If $p$ and $h$ satisfy these conditions, we say that $h$ is a Sylvester form for $p$. Theorem 2.3 will only be used in cases where $a_{j}, \alpha_{j}, \beta_{j} \in \mathbb{R}$. We remark that the $\lambda_{k}$ 's are found by solving (2.3), a system of linear equations with real coefficients: the existence of a solution over $\mathbb{C}$ implies the existence of a solution over $\mathbb{R}$.

Another useful classical result is in the first article [11] published in the Proceedings of the London Mathematical Society. Sylvester proved a conjecture of Newton regarding the number of complex zeros of certain real polynomials. This is presented with proof by Pólya and Szegö in [4, p. 48, Prob. 79].

Theorem 2.4 (Sylvester). Suppose $0 \neq \lambda_{k}$ for all $k$ and $\gamma_{1}<\cdots<\gamma_{r}, r \geq 2$, are real numbers such that

$$
Q(t)=\sum_{k=1}^{r} \lambda_{k}\left(t-\gamma_{k}\right)^{d}
$$

does not vanish identically. Suppose the sequence $\left(\lambda_{1}, \ldots, \lambda_{r},(-1)^{d} \lambda_{1}\right)$ has $C$ changes of sign and $Q$ has $Z$ zeros, counting multiplicity. Then $Z \leq C$.

We shall apply a homogenized version of this result. 
Corollary 2.5. Suppose $p(x, y)$ is a non-zero real form of degree $d$ with $\tau$ real linear factors (counting multiplicity) and

$$
p(x, y)=\sum_{k=1}^{r} \lambda_{k}\left(\cos \theta_{k} x-\sin \theta_{k} y\right)^{d},
$$

where $-\frac{\pi}{2}<\theta_{1}<\cdots<\theta_{r} \leq \frac{\pi}{2}, r \geq 2$, and $\lambda_{k} \neq 0$ for all $k$. Suppose there are $\sigma$ sign changes in the sequence $\left(\lambda_{1}, \lambda_{2}, \ldots, \lambda_{r},(-1)^{d} \lambda_{1}\right)$. Then $\tau \leq \sigma$.

Proof. We first "projectivize" (2.5) to get the following representation for $p$ :

$$
\sum_{k=1}^{r} \frac{\lambda_{k}}{2}\left(\cos \theta_{k} x-\sin \theta_{k} y\right)^{d}+\sum_{k=1}^{r}(-1)^{d} \frac{\lambda_{k}}{2}\left(\cos \left(\theta_{k}+\pi\right) x-\sin \left(\theta_{k}+\pi\right) y\right)^{d} .
$$

Observe that the sequence $\frac{1}{2}\left(\lambda_{1}, \lambda_{2}, \ldots, \lambda_{r},(-1)^{d} \lambda_{1},(-1)^{d} \lambda_{2}, \ldots,(-1)^{d} \lambda_{r}, \lambda_{1}\right)$ has $2 \sigma$ sign changes, as does any cyclic permutation thereof. Thus in taking any block of $r$ (cyclically) consecutive summands from (2.6) as "the" representation (2.5), $\sigma$ will be unchanged. Accordingly, we may make an invertible change of variables

$$
(x, y) \mapsto(\cos \theta x+\sin \theta y,-\sin \theta x+\cos \theta y)
$$

in (2.5) to "dial" the angles as we wish without changing $\tau$ and $\sigma$. We do so to choose $r$ consecutive angles in the interval $\left(-\frac{\pi}{2}, \frac{\pi}{2}\right)$ and so that $y$ does not divide $p$.

Having done so, we now dehomogenize (2.5) into

$$
Q(t):=p(t, 1)=\sum_{k=1}^{r} \lambda_{k} \cos ^{d} \theta_{k}\left(t-\tan \theta_{k}\right)^{d} .
$$

Since $y \nmid p(x, y)$, there are no "zeros at infinity" and by Theorem 2.4, the number of linear factors of $p$ is equal to the number of zeros of $Q: \tau=Z \leq C=\sigma$.

For a completely self-contained proof of Corollary 2.5, see 8]. Note that both Theorem 2.3 and Corollary 2.5 apply whether $d$ is even or odd and that Corollary 2.5 applies to irregular representations of $\ell^{2 s}$ such as (1.2). We shall improve the following corollary in Theorem 3.1.

Corollary 2.6. If $p(x, y) \neq \pm \ell^{2 s}$ splits as a product of $2 s$ real linear forms and $(a, b) \in \mathcal{B}(p)$, then $(s, s) \preceq(a, b)$.

Proof. By Theorem 2.1, $p$ has at least one representation, necessarily of length $\geq 2$, which can be put into the form of (2.5). Since there are at least $2 s$ sign changes, it must have at least $s$ positive coefficients and $s$ negative coefficients.

The final tool involves earlier work $[6$ of the author. Define

$$
Q_{n, 2 s}^{r}=\left\{\sum_{k=1}^{r}\left(\xi_{k 1} x_{1}+\cdots+\xi_{k n} x_{n}\right)^{2 s}: \xi_{k j} \in \mathbb{R}\right\}, \quad Q_{n .2 s}=\bigcup_{r=1}^{\infty} Q_{n, 2 s}^{r} .
$$

The elements of $Q_{n, 2 s}^{r}$ have representations (1.1) in which $\lambda_{k}>0$ for all $k$. Equivalently, $p \in Q_{n, 2 s}^{r}$ if and only if $(t, 0) \in \mathcal{B}(p)$ for some integer $t \leq r$.

Theorem 2.7 ([6, pp. 36-37]). For any $(n, 2 s, r), Q_{n, 2 s}^{r}$ is a closed set and $Q_{n, 2 s}$ is a closed convex cone. 
The topology here is the usual one, of coefficientwise convergence. There is a classical inner product on forms under which the dual cone to $Q_{n, 2 s}$ is $P_{n, 2 s}$, the cone of positive semidefinite forms of degree $2 s$ in $n$ variables. (See [6, 7] for an extensive discussion of this inner product.) Let $\Sigma_{n, 2 s}$ denote the cone of sums of squares of polynomials of degree $s$. Hilbert proved that $\Sigma_{n, 2 s}=P_{n, 2 s}$ only for $n=2,2 s=2$ and $(n, 2 s)=(3,4)$. In these cases, the dual cone to $Q_{n, 2 s}$ is $\Sigma_{n, 2 s}$, affording a decisive condition for membership in $Q_{n, 2 s}$.

We restrict our attention to $F_{2,2 s}$. We associate to $p(x, y)=\sum_{j=0}^{2 s}\left(\begin{array}{c}2 s \\ j\end{array}\right) a_{j} x^{2 s-j} y^{j}$ the catalecticant quadratic form

$$
H_{p}(t)=H_{p}\left(t_{0}, \ldots, t_{s}\right):=\sum_{i=0}^{s} \sum_{j=0}^{s} a_{i+j} t_{i} t_{j}
$$

(The matrix for $H_{p}$ corresponds to $(d, r)=(2 s, s)$ in (2.4).) Observe that

$$
p(x, y)=\sum_{k=1}^{r} \lambda_{k}\left(\alpha_{k} x+\beta_{k} y\right)^{2 s} \Longleftrightarrow H_{p}(t)=\sum_{k=1}^{r} \lambda_{k}\left(\sum_{i=0}^{s} \alpha_{k}^{s-i} \beta_{k}^{i} t_{i}\right)^{2} .
$$

If $f(x, y)=\sum_{i}\left(\begin{array}{c}d \\ i\end{array}\right) a_{i} x^{d-i} y^{i}$ and $g(x, y)=\sum_{i}\left(\begin{array}{c}d \\ i\end{array}\right) b_{i} x^{d-i} y^{i}$, then the inner product is defined to be $[f, g]=\sum_{i}\left(\begin{array}{c}d \\ i\end{array}\right) a_{i} b_{i}$. In particular, if $L\left(t_{0}, \ldots, t_{n} ; x, y\right)=$ $\sum_{i=0}^{s} t_{i} x^{s-i} y^{i}$, then $H_{p}(t)=\left[p, L^{2}\right]$.

Lemma 2.8. If $p \in F_{2,2 s}, \alpha \delta-\beta \gamma \neq 0$ and $q(x, y)=p(\alpha x+\beta y, \gamma x+\delta y)$, then $S\left(H_{q}\right)=S\left(H_{p}\right)$.

Proof. Define $\tilde{t}_{i}$ by

$$
\sum_{i=0}^{s} \tilde{t}_{i} x^{s-i} y^{i}=\sum_{i=0}^{s} t_{i}(\alpha x+\gamma y)^{s-i}(\beta x+\delta y)^{i}
$$

the $\tilde{t}_{i}$ 's are linear functions of the $t_{i}$ 's. Now let

$$
\tilde{L}\left(t_{0}, \ldots, t_{s} ; x, y\right):=L\left(t_{0}, \ldots, t_{s} ; \alpha x+\gamma y, \beta x+\delta y\right)=L\left(\tilde{t}_{0}, \ldots, \tilde{t}_{n} ; x, y\right) .
$$

By the contravariant property of the inner product ([6, Thm. 2.15]), $H_{q}(t)=\left[q, L^{2}\right]$ $=\left[p, \tilde{L}^{2}\right]=H_{p}(\tilde{t})$, so that $S\left(H_{q}\right) \preceq S\left(H_{p}\right)$. The change of variables from $p$ to $q$ is invertible, so $S\left(H_{p}\right) \preceq S\left(H_{q}\right)$ as well.

Theorem 2.9 ([6, pp. 41, 61]). Suppose $p \in F_{2,2 s}$ and $\operatorname{rank}\left(H_{p}\right)=w$.

(1) We have $p \in Q_{2,2 s}$ if and only if $H_{p}(t)$ is positive semidefinite.

(2) If $p \in Q_{2,2 s}$ and $w \leq s$, then $p$ has only one honest representation as a sum of $2 s$-th powers; this representation has $w$ summands, and so $(w, 0) \in \mathcal{B}(p)$.

(3) If $p \in Q_{2,2 s}$ and $w=s+1$, then $p$ cannot be written as a sum of $s$ or fewer $2 s$-th powers. Furthermore, for each $(\alpha, \beta) \neq(0,0)$, there exists $\lambda>0$ so that $p$ can be written as a sum of $s+12 s$-th powers, one of which is $\lambda(\alpha x+\beta y)^{2 s}$, and so $(s+1,0) \in \mathcal{B}(p)$.

If $p \in Q_{2,2 s}$, then $w=\operatorname{rank}\left(H_{p}\right)$ is called the width of $p$. Uniqueness in Theorem $2.9(2)$ is only asserted within the class of representations with positive coefficients; cf. (1.2).

Corollary 2.10. Suppose $p \in F_{2,2 s}$ and suppose $S\left(H_{p}\right)=\left(p_{H}, n_{H}\right)$.

(1) If $(a, b) \in \mathcal{B}(p)$, then $\left(p_{H}, n_{H}\right) \preceq(a, b)$. 
(2) If $p \in Q_{2,2 s}$, then $S(p)=S\left(H_{p}\right)=\left(p_{H}, n_{H}\right)=\left(\operatorname{rank}\left(H_{p}\right), 0\right)$.

(3) If $(a, b) \in \mathcal{B}(p)$ is such that $a+b=p_{H}+n_{H}=\operatorname{rank}\left(H_{p}\right)$, then the signature of $p$ is $S(p)=S\left(H_{p}\right)$.

Proof. If $p$ has a representation with badge $(a, b)$, then (2.8) implies that $H_{p}$ has a representation with the same badge. Quadratic forms have unique signatures by Theorem 2.1, so (1) is immediate. If $p \in Q_{2,2 s}$, then by parts (2) and (3) of Theorem 2.9, $\left(\operatorname{rank}\left(H_{p}\right), 0\right) \in \mathcal{B}(p)$; hence by $(1)$ it is the unique minimal element. Finally if $(a, b) \in \mathcal{B}(p)$ with $a+b=\operatorname{rank}\left(H_{p}\right)$, then (1) implies that $(a, b)=\left(r_{H}, n_{H}\right)$ and $(a, b)$ is the unique minimal badge in $\mathcal{B}(p)$.

The practical significance of Corollary 2.10(3) is that Theorem 2.3 might already show that the length of $p$ is equal to the rank of $H_{p}$. In such a case, we know that the signature of $p$ is $S\left(H_{p}\right)$ without having to compute an actual representation.

If $s>1$, then $\left(p_{H}, n_{H}\right)$ need not belong to $\mathcal{B}(p)$. By Corollary 2.6, if $p \neq \pm \ell^{2 s}$ splits into $2 s$ linear factors, then any $(a, b) \in \mathcal{B}(p)$ will have $a+b \geq 2 s$, but $r_{H}+n_{H} \leq s+1$.

We also remark (see [6, p. 124]) that $\left(x^{2}+y^{2}\right)^{s}$ always has width $s+1$, and so has signature $(s+1,0)$. Theorem $2.9(3)$ is illustrated by the identity

$$
\left(\begin{array}{c}
2 s \\
s
\end{array}\right)\left(x^{2}+y^{2}\right)^{s}=\frac{1}{s+1} \sum_{k=0}^{s}\left(\cos \left(\frac{k \pi}{s+1}+\theta\right) x+\sin \left(\frac{k \pi}{s+1}+\theta\right) y\right)^{2 s}, \quad \theta \in \mathbb{R} .
$$

Finally, it is easy to construct jump signature sequences in $Q_{n, 2 s}$ and $F_{n, 2}$ in which the jump is down. If

$$
f_{m}=x^{2 s}+\frac{1}{m}\left(x^{2}+y^{2}\right)^{s},
$$

then $S\left(f_{m}\right)=(s+1,0)$ and $f_{m} \rightarrow f$, where $S(f)=(1,0)$. Since each $Q_{2,2 s}^{r}$ is closed, any jump in $Q_{2,2 s}$ will be down.

For quadratic forms, only downward jumps are possible, but every feasible downward jump occurs. Suppose $a_{i}, b_{i} \geq 0$ and $a_{1}+a_{2}+b_{1}+b_{2} \leq n$. Let

$$
f_{m}\left(x_{1}, \ldots, x_{n}\right)=\sum_{i=1}^{a_{1}} x_{i}^{2}+\frac{1}{m} \sum_{i=a_{1}+1}^{a_{1}+a_{2}} x_{i}^{2}-\sum_{i=a_{1}+a_{2}+1}^{a_{1}+a_{2}+b_{1}} x_{i}^{2}-\frac{1}{m} \sum_{i=a_{1}+a_{2}+b_{1}+1}^{a_{1}+a_{2}+b_{1}+b_{2}} x_{i}^{2} .
$$

Then $S\left(f_{m}\right)=\left(a_{1}+a_{2}, b_{1}+b_{2}\right)$ and $f_{m} \rightarrow f$, where $S(f)=\left(a_{1}, b_{1}\right)$. On the other hand, for $f \in F_{n, 2}, S(f)$ is the number of positive and negative roots (counting multiplicity) of $\phi_{f}(t)$, the characteristic polynomial of the matrix associated to $f$. Since all roots of $\phi_{f}(t)$ are real and since the roots of a polynomial are continuous functions of its coefficients, it follows that if $S\left(f_{m}\right)=(a, b)$ and $f_{m} \rightarrow f$, then $S(f) \preceq(a, b)$.

\section{Signatures}

In this section, we determine all possible signatures in $F_{2,2 s}$ and we find some restrictions on incomparable signatures. Let $[[0, s]]^{2}=\left\{(i, j) \in \mathbb{Z}^{2}: 0 \leq i, j \leq s\right\}$.

Theorem 3.1. Fix $s \geq 1$.

(1) If $p \in F_{2,2 s}$, then $\mathcal{S}(p) \subseteq\{(s+1,0),(0, s+1)\} \cup[[0, s]]^{2}$.

(2) If $p(x, y) \neq \pm \ell^{2 s}$ is a product of $2 s$ real linear factors, then $S(p)=(s, s)$.

(3) If $(u, v) \in\{(s+1,0),(0, s+1)\} \cup[[0, s]]^{2}$, then there exists $p \in F_{2,2 s}$ such that $S(p)=(u, v)$. 
Proof. Suppose $(a, b) \in \mathcal{S}(p)$ and, specifically, suppose that

$$
p(x, y)=\sum_{i=1}^{a}\left(\alpha_{i} x+\beta_{i} y\right)^{2 s}-\sum_{j=1}^{b}\left(\gamma_{j} x+\delta_{j} y\right)^{2 s}:=p_{+}(x, y)-p_{-}(x, y) .
$$

Since (3.1) is honest and $p_{+}, p_{-} \in Q_{2,2 s}$, Theorem 2.9 implies that $p_{+}$and $p_{-}$can each be written as a sum of $\leq s+12 s$-th powers. Since $(a, b)$ is minimal, $a, b \leq s+1$.

We now show that if $(s+1, b) \in \mathcal{S}(p)$, then $b=0$. If so, by Theorem 2.9(3), we can rewrite $p_{+}$as a sum of length $s+1$, one of whose summands is $\lambda\left(\gamma_{1} x+\delta_{1} y\right)^{2 s}$. This cancels with the summand $\left(\gamma_{1} x+\delta_{1} y\right)^{2 s}$ in $p_{-}$to yield a representation with a smaller badge. A similar contradiction results from $(a, s+1)$ with $a>0$.

For (2), combine Corollary 2.6 and part (1).

For (3), first note that $p(x, y)= \pm\left(x^{2}+y^{2}\right)^{s}$ has signature $(s+1,0)$ or $(0, s+1)$. We now show that every $(u, v) \in[[0, s]]^{2}$ occurs. If $\psi$ is any product of $2 s$ distinct linear factors, then $S(\psi)=(s, s)$ by $(2)$, and there exists a representation

$$
\psi(x, y)=\sum_{j=1}^{s}\left(\alpha_{j} x+\beta_{j} y\right)^{2 s}-\sum_{j=1}^{s}\left(\gamma_{j} x+\delta_{j} y\right)^{2 s} .
$$

We claim that for $0 \leq u, v \leq s$,

$$
\psi_{u, v}(x, y)=\sum_{j=1}^{u}\left(\alpha_{j} x+\beta_{j} y\right)^{2 s}-\sum_{j=1}^{v}\left(\gamma_{j} x+\delta_{j} y\right)^{2 s}
$$

has a unique signature $(u, v)$. If $(\tilde{u}, \tilde{v})$ is another badge for $\psi_{u, v}$ and

$$
\psi_{u, v}(x, y)=\sum_{j=1}^{\tilde{u}}\left(\tilde{\alpha}_{j} x+\tilde{\beta}_{j} y\right)^{2 s}-\sum_{j=1}^{\tilde{v}}\left(\tilde{\gamma}_{j} x+\tilde{\delta}_{j} y\right)^{2 s},
$$

then we may add the "missing" summands to reconstitute $\psi$ :

$\psi(x, y)=\sum_{j=1}^{\tilde{u}}\left(\tilde{\alpha}_{j} x+\tilde{\beta}_{j} y\right)^{2 s}+\sum_{j=u+1}^{s}\left(\alpha_{j} x+\beta_{j} y\right)^{2 s}-\sum_{j=1}^{\tilde{v}}\left(\tilde{\gamma}_{j} x+\tilde{\delta}_{j} y\right)^{2 s}-\sum_{j=v+1}^{s}\left(\gamma_{j} x+\delta_{j} y\right)^{2 s}$.

Since $(s, s) \preceq(\tilde{u}+s-u, \tilde{v}+s-v)$, we have $(u, v) \preceq(\tilde{u}, \tilde{v})$, as claimed.

Example 3.1. The representation

$$
p(x, y)=8 x^{4}+48 x^{2} y^{2}-8 y^{4}=(x+2 y)^{4}+6 x^{4}+(x-2 y)^{4}-40 y^{4} \in F_{2,4}
$$

shows that $p$ has badge $(3,1)$, which cannot be minimal by Theorem 3.1(1). Using the argument of the proof, rewrite $p_{+}$using (1.2) and cancel terms:

$$
p(x, y)=4(x+y)^{4}+4(x-y)^{4}+24 y^{4}-40 y^{4}=4(x+y)^{4}+4(x-y)^{4}-16 y^{4},
$$

so $(2,1) \in \mathcal{B}(p)$. Since $H_{p}\left(t_{0}, t_{1}, t_{2}\right)=8\left(t_{0}^{2}+t_{0} t_{2}-t_{2}^{2}+t_{1}^{2}\right)$ has signature $(2,1)$, it follows from Corollary 2.10 that $S(p)=(2,1)$.

We now give a necessary condition for incomparable signatures.

Theorem 3.2. Suppose $p \in F_{2,2 s}$ has two signatures $(a, b)$ and $(c, d)$ such that $a>c$ and $b<d$. Then $a+d \geq s+3, b+c \geq s+1, \max \{a+b, c+d\} \geq s+2$ and $a, b, c, d \geq 1$. 
Proof. Write

$$
\begin{aligned}
& p=p_{+}-p_{-}=\sum_{i=1}^{a}\left(\alpha_{i} x+\beta_{i} y\right)^{2 s}-\sum_{i=1}^{b}\left(\gamma_{i} x+\delta_{i} y\right)^{2 s}, \\
& p=\tilde{p}_{+}-\tilde{p}_{-}=\sum_{i=1}^{c}\left(\tilde{\alpha}_{i} x+\tilde{\beta}_{i} y\right)^{2 s}-\sum_{i=1}^{d}\left(\tilde{\gamma}_{i} x+\tilde{\delta}_{i} y\right)^{2 s} .
\end{aligned}
$$

We obtain two representations for $q:=p_{+}+\tilde{p}_{-}=\tilde{p}_{+}+p_{-}$:

$$
\begin{aligned}
& q(x, y)=\sum_{i=1}^{a}\left(\alpha_{i} x+\beta_{i} y\right)^{2 s}+\sum_{i=1}^{d}\left(\tilde{\gamma}_{i} x+\tilde{\delta}_{i} y\right)^{2 s}, \\
& q(x, y)=\sum_{i=1}^{b}\left(\gamma_{i} x+\delta_{i} y\right)^{2 s}+\sum_{i=1}^{c}\left(\tilde{\alpha}_{i} x+\tilde{\beta}_{i} y\right)^{2 s} .
\end{aligned}
$$

Observe that $q \in Q_{2,2 s}$, and suppose it has width $w$. By Theorem 2.9, $q$ has only one honest representation as a sum of $2 s$-th powers, and it has $w$ summands. The representations for $q$ in (3.3) might not be honest, because they combine two different honest sums and proportional summands might occur in both.

Suppose first that $w \leq s$. Since each $\left(\alpha_{i} x+\beta_{i} y\right)^{2 s}$ occurs in the first representation for $q$, some multiple must occur in the second representation. The representations in (3.2) are honest, so this multiple cannot be one of the $\left(\gamma_{i} x+\delta_{i} y\right)^{2 s}$ 's, and so must be $\left(\tilde{\alpha}_{k} x+\tilde{\beta}_{k} y\right)^{2 s}$ for some $k$. But the $\left(\alpha_{i} x+\beta_{i} y\right)^{2 s}$ 's are distinct, as are the $\left(\tilde{\alpha}_{i} x+\tilde{\beta}_{i} y\right)^{2 s}$ 's, since they come from honest sums. Thus, $a \leq c$, a contradiction.

We must therefore have that $w=s+1$, and hence $b+c \geq s+1$; and since $a \geq c+1$ and $d \geq b+1$, we have $a+d \geq s+3$ and so $(a+b)+(c+d)=(a+d)+(b+c) \geq 2 s+4$. Finally, $a, d \geq 1$ by hypothesis. If $b=0$, then $c \geq s+1$, which contradicts $a>c$ by Theorem 3.1(1). Assuming $c=0$ leads to a similar contradiction.

\section{Quartics AND SEXTICS}

We begin this section with an application of the last two theorems.

Theorem 4.1. A binary quartic form has a unique signature.

Proof. By Theorem 3.1(1), the possible signatures in $F_{2,4}$ are $(0,3),(3,0)$ and $[[0,2]]^{2}$. By Theorem 3.2, if $p$ has two incomparable signatures $(a, b)$ and $(c, d)$, then $a+b+c+d \geq 5+3=8$, so $(a, b)=(c, d)=(2,2)$, a contradiction.

The uniqueness allows us to compute the signatures of binary quartics.

Theorem 4.2. If $p \in F_{2,4}$, then either $S(p)=S\left(H_{p}\right)$ or $p \neq \pm \ell^{4}$ is a product of linear factors and $S(p)=(2,2)$.

Proof. For clarity we write out $p$ and the matrix for $H_{p}$ :

$$
p(x, y)=a_{0} x^{4}+4 a_{1} x^{3} y+6 a_{2} x^{2} y^{2}+4 a_{3} x y^{3}+a_{4} y^{4}, \quad H_{p}=\left(\begin{array}{ccc}
a_{0} & a_{1} & a_{2} \\
a_{1} & a_{2} & a_{3} \\
a_{2} & a_{3} & a_{4}
\end{array}\right) .
$$

If $p$ is a product of real linear factors, then either $p= \pm(\alpha x+\beta y)^{4}$, in which case $S(p)=S\left(H_{p}\right)=(1,0)$ or $(0,1)$ or else $S(p)=(2,2)$ by Theorem $3.1(2)$. 
Otherwise, we may assume that $p$ has a definite quadratic factor. By Lemma 2.8, neither $S(p)$ nor $S\left(H_{p}\right)$ is affected by an invertible linear change of variables. Therefore, we may diagonalize this factor and assume that

$$
p(x, y)=\left(x^{2}+y^{2}\right)\left(a x^{2}+b x y+c y^{2}\right) .
$$

Now apply (2.7) and, in effect, rotate the axes so that $x^{2}+y^{2}$ is unchanged and $a x^{2}+b x y+c y^{2}$ loses its $x y$-term. After possible scaling, multiplying by -1 (which flips both $S(p)$ and $S\left(H_{p}\right)$ ) and permuting $x$ and $y$, we see that $p$ can be written as

$$
q_{u}(x, y)=\left(x^{2}+y^{2}\right)\left(x^{2}+u y^{2}\right)=x^{4}+6\left(\frac{u+1}{6}\right) x^{2} y^{2}+y^{4}, \quad|u| \leq 1 .
$$

As noted earlier, $S\left(q_{1}\right)=S\left(H_{q_{1}}\right)=(3,0)$, and as $q_{-1}(x, y)=x^{4}-y^{4}$ and $H_{q_{-1}}=$ $t_{0}^{2}-t_{2}^{2}, S\left(q_{-1}\right)=S\left(H_{q_{-1}}\right)=(1,1)$. Assume $|u|<1$, let $\rho^{2}=\frac{u+1}{6}$ and note that

$$
q_{u}(x, y)=\frac{1}{2}\left((x+\rho y)^{4}+(x-\rho y)^{4}\right)+\left(\frac{-1+34 u-u^{2}}{36}\right) y^{4} .
$$

Since $u \in(-1,1)$, (4.1) has badge $(3,0)$ if $u>17-12 \sqrt{2},(2,0)$ if $u=17-12 \sqrt{2}$ and $(2,1)$ if $u<17-12 \sqrt{2}$. On the other hand,

$$
H_{q_{u}}=\left(\begin{array}{ccc}
1 & 0 & \frac{1+u}{6} \\
0 & \frac{1+u}{6} & 0 \\
\frac{1+u}{6} & 0 & u
\end{array}\right),
$$

and since $\frac{1+u}{6}>0$ for $u \in(-1,1), S\left(H_{q}\right)$ is determined by the sign of $u-\left(\frac{1+u}{6}\right)^{2}=$ $\frac{-1+34 u-u^{2}}{36}$. In each case $S\left(q_{u}\right)=S\left(H_{q_{u}}\right)$, and this completes the proof.

The quartic $q_{17-12 \sqrt{2}}(x, y)$ is less mysterious than it seems:

$$
\begin{gathered}
x^{4}+y^{4}=\left(x^{2}+\sqrt{2} x y+y^{2}\right)\left(x^{2}-\sqrt{2} x y+y^{2}\right) \mapsto \\
\left(u^{2}+v^{2}\right)\left(u^{2}-4 u v+5 v^{2}\right) \mapsto\left(w^{2}+z^{2}\right)\left((3+2 \sqrt{2}) w^{2}+(3-2 \sqrt{2}) z^{2}\right) \\
=(3+2 \sqrt{2}) q_{17-12 \sqrt{2}}(w, z)
\end{gathered}
$$

under $(x, y) \mapsto(u-v, \sqrt{2} v)$ and $(u, v) \mapsto(a w+b z,-b w+a z)$, where $a=\cos \frac{3 \pi}{8}=$ $\frac{1}{4}(2-\sqrt{2})^{1 / 2}$ and $b=\sin \frac{3 \pi}{8}=\frac{1}{4}(2+\sqrt{2})^{1 / 2}$. Another approach to real canonical forms for real quartics can be found in [5, p. 217].

Example 4.1. The only possible positive jumps in $F_{2,4}$ occur if $f_{m} \rightarrow f$ and $S(f)=(2,2)$, since otherwise $S(p)=S\left(H_{p}\right)$. For example, let

$$
f_{m}(x, y)=\frac{1}{m} x^{4}+6 x^{2} y^{2}+\frac{1}{m} y^{4}=\frac{1}{m}\left(x^{4}+6 m x^{2} y^{2}+y^{4}\right) .
$$

It is easy to see that for $m \geq 1, S\left(f_{m}\right)=(2,1)$, but $f_{m} \rightarrow f$, where $f(x, y)=x^{2} y^{2}$ and $S(f)=(2,2)$. In other words, $Q_{2,4}^{2}-Q_{2,4}^{1}$ is not a closed set.

We turn to sextics. Theorem 3.2 does not rule out incomparable signatures.

Corollary 4.3. If $p \in S_{2,6}$ and $|\mathcal{S}(p)| \geq 2$, then $\mathcal{S}(p)=\{(2,3),(3,2)\}$.

Proof. If $(a, b)$ and $(c, d)$ are signatures for a sextic and $a>c, b<d$, then Theorem 3.2 implies that $a+d \geq 6, b+c \geq 4$ and so $a=d=3$ and $b=c=2$.

The following theorem analyzes our principal binary sextic example.

Theorem 4.4. Let $q_{\lambda}(x, y)=6 x^{5} y+20 \lambda x^{3} y^{3}+6 x y^{5}$.

(1) If $\lambda=1$, then $\mathcal{S}\left(q_{\lambda}\right)=\{(1,1)\}$. 
(2) If $\lambda>0, \lambda \neq 1$, then $\mathcal{S}\left(q_{\lambda}\right)=\{(2,2)\}$.

(3) If $-\frac{3}{5}<\lambda \leq 0$, then $\mathcal{S}\left(q_{\lambda}\right)=\{(2,3),(3,2)\}$.

(4) If $\lambda \leq-\frac{3}{5}$, then $\mathcal{S}\left(q_{\lambda}\right)=\{(3,3)\}$.

Before we prove this theorem, we state its immediate corollary.

Corollary 4.5. The Law of Inertia fails for binary sextics: there exists $q \in F_{2,6}$ with two signatures.

The following lemma is a fruitful way of generating forms with two signatures.

Lemma 4.6. If $p(x,-y)=-p(x, y)$, then $(a, b) \in \mathcal{B}(p) \Longrightarrow(b, a) \in \mathcal{B}(p)$.

Proof. Since $p(x, y)=-p(x,-y)$,

$$
p(x, y)=\sum_{k=1}^{r} \lambda_{k}\left(\alpha_{k} x+\beta_{k} y\right)^{2 s} \Longrightarrow p(x, y)=\sum_{k=1}^{r}-\lambda_{k}\left(\alpha_{k} x-\beta_{k} y\right)^{2 s} .
$$

Proof of Theorem 4.4. We first note that $q_{\lambda}(x, y)=6 x y\left(x^{4}+\frac{10}{3} \lambda x^{2} y^{2}+y^{4}\right)$ is a product of six linear factors if $\frac{10}{3} \lambda \leq-2$, establishing case (4). The eigenvalues of

$$
H_{q_{\lambda}}=\left(\begin{array}{cccc}
0 & 1 & 0 & \lambda \\
1 & 0 & \lambda & 0 \\
0 & \lambda & 0 & 1 \\
\lambda & 0 & 1 & 0
\end{array}\right)
$$

are $\pm(1+\lambda), \pm(1-\lambda)$. Thus, if $\lambda \neq \pm 1$, then $S\left(H_{q_{\lambda}}\right)=(2,2)$. If $\lambda=1$, then $S\left(H_{q_{1}}\right)=(1,1)$ and $q_{1}(x, y)=\frac{1}{2}(x+y)^{6}-\frac{1}{2}(x-y)^{6}$, establishing case (1). If $\lambda>-\frac{3}{5}, \lambda \neq 1$, then it follows from Corollary 2.10, Theorem 3.1(1) and Lemma 4.6 that $\mathcal{S}\left(q_{\lambda}\right)$ is $\{(2,2)\},\{(2,3),(3,2)\}$ or $\{(3,3)\}$, depending only on the length of $q_{\lambda}$.

Suppose $\lambda \neq 1, \lambda>-\frac{3}{5}$ and $q_{\lambda}$ has length 4 . Then $h(x, y)=\sum_{i=0}^{4} c_{i} x^{4-i} y^{i}$ is a Sylvester form for $q_{\lambda}$ provided it has four distinct real factors and

$$
\left(\begin{array}{ccccc}
0 & 1 & 0 & \lambda & 0 \\
1 & 0 & \lambda & 0 & 1 \\
0 & \lambda & 0 & 1 & 0
\end{array}\right) \cdot\left(\begin{array}{l}
c_{0} \\
c_{1} \\
c_{2} \\
c_{3} \\
c_{4}
\end{array}\right)=\left(\begin{array}{l}
0 \\
0 \\
0
\end{array}\right)
$$

Since $c_{1}+\lambda c_{3}=\lambda c_{1}+c_{3}=0$ and $\lambda^{2} \neq 1, h(x, y)=c_{0} x^{4}+c_{2} x^{2} y^{2}-\left(c_{0}+\lambda c_{2}\right) y^{4}$. If $c_{0}=0$ or $c_{0}+\lambda c_{2}=0$, then $h$ is divisible by $y^{2}$ or $x^{2}$; hence we may scale so that $c_{0}=1$ and assume $h(x, y)=\left(x^{2}-\gamma_{1}^{2} y^{2}\right)\left(x^{2}-\gamma_{2}^{2} y^{2}\right)$ for some real $\gamma_{1}, \gamma_{2} \neq 0$. In this case, $c_{2}=-\left(\gamma_{1}^{2}+\gamma_{2}^{2}\right)$ and

$$
\gamma_{1}^{2} \gamma_{2}^{2}=-1+\lambda\left(\gamma_{1}^{2}+\gamma_{2}^{2}\right)
$$

This is clearly impossible if $\lambda \leq 0$. If $\lambda>0$, then (4.2) is equivalent to

$$
\gamma_{2}^{2}=\frac{\lambda \gamma_{1}^{2}-1}{\gamma_{1}^{2}-\lambda}
$$

If $\lambda>1$, then $\gamma_{1}^{2}=2 \lambda$ implies $\gamma_{2}^{2}=2 \lambda-1 / \lambda>0$; if $\lambda<1$, then $\gamma_{1}^{2}=2 / \lambda$ implies $\gamma_{2}^{2}=\frac{\lambda}{2-\lambda^{2}}$ and $\gamma_{1}^{2}>2>\lambda>\gamma_{2}^{2}$. In either case, $h$ is a Sylvester form, so $q_{\lambda}$ has length 4 , and by Corollary 2.10(3), $S\left(q_{\lambda}\right)=(2,2)$. This establishes $(2)$. 
In the remaining case, $-\frac{3}{5}<\lambda \leq 0$. We wish to find a quintic Sylvester form $h_{\lambda}(x, y)=\sum_{i=0}^{5} c_{i} x^{5-i} y^{i}$. Take $0<u<v$ and let

$$
\begin{gathered}
h_{\lambda}(x, y)=(x+y)\left(x^{2}+(2+u) x y+y^{2}\right)\left(x^{2}+(2+v) x y+y^{2}\right), \\
6+u+v+\lambda(10+3 u+3 v+u v)=0 .
\end{gathered}
$$

This satisfies the system

$$
\left(\begin{array}{llllll}
0 & 1 & 0 & \lambda & 0 & 1 \\
1 & 0 & \lambda & 0 & 1 & 0
\end{array}\right) \cdot\left(\begin{array}{l}
c_{0} \\
c_{1} \\
c_{2} \\
c_{3} \\
c_{4} \\
c_{5}
\end{array}\right)=\left(\begin{array}{l}
0 \\
0
\end{array}\right) .
$$

By setting $v=2 u$, we see that (4.3) holds for $u=\frac{3+5 \lambda}{-2 \lambda}$ and $v=\frac{3+5 \lambda}{-\lambda}$, so that $q_{\lambda}$ for $\lambda \in\left(-\frac{3}{5}, 0\right)$ has length five and $S\left(q_{\lambda}\right)=\{(2,3),(3,2)\}$. For $\lambda=0$, (4.3) is impossible for positive $u, v$. In this case, (4.4) becomes $c_{0}+c_{4}=c_{1}+c_{5}=0$. Let

$$
g(x, y)=x(x+y)(x+2 y)(x+3 y)(x-6 y)=x^{5}-25 x^{3} y^{2}-60 x^{2} y^{3}-36 x y^{4}
$$

and let $h_{0}(x, y)=g(x, y / \sqrt{6})$. Then $c_{0}=1, c_{4}=-6^{-2} \cdot 36=-1$ and $c_{1}=c_{5}=0$, so that $h_{0}$ satisfies (4.4) and $h_{0}$ has length five, so $S\left(q_{0}\right)=\{(2,3),(3,2)\}$.

Example 4.2. An exact calculation shows that

$$
\begin{gathered}
1296(x+y)^{6}-567(x+2 y)^{6}+112(x+3 y)^{6}-(x-6 y)^{6}-840 x^{6} \\
=3024\left(x^{5} y+36 x y^{5}\right) .
\end{gathered}
$$

If we scale (4.5) by sending $y \mapsto \frac{y}{\sqrt{6}}$ and divide by $84 \sqrt{6}$, we obtain a representation of $q_{0}(x, y)=6 x^{5} y+6 x y^{5}$. Observe that $\lim _{m} q_{1 / m} \rightarrow q_{0}$ provides another example of a positive jump. Also observe that for $\lambda$ close to 0 , neither $S\left(H_{q_{\lambda}}\right)$ nor the zero structure of $q_{\lambda}$ change, but a jump occurs.

Larger jumps are possible, as shown in our final example.

Example 4.3. Let $r_{\lambda}(x, y)=\left(x^{2}-y^{2}\right)^{3}+15 \lambda x^{2} y^{2}\left(x^{2}-y^{2}\right)$. Then $r_{0}(x, y)$ is a product of six linear factors, so $S\left(r_{0}\right)=(3,3)$ and $r_{1 / 5}(x, y)=x^{6}-y^{6}$, so $S\left(r_{1 / 5}\right)=$ $(1,1)$. We now show that if $0<\lambda<\frac{1}{5}$, then $S\left(r_{\lambda}\right)=(2,2)$. First, it is easy to show that $S\left(H_{r_{\lambda}}\right)=(2,2)$ if $\lambda \neq 0,-\frac{4}{5}$; hence the length of $r_{\lambda}$ is $\geq 4$ if $\lambda \in\left(0, \frac{1}{5}\right)$. Second, we produce a Sylvester form of degree four: $h(x, y)=\sum_{i=0}^{4} c_{i} x^{4-i} y^{i}$ must have distinct real factors and satisfy

$$
\left(\begin{array}{ccccc}
1 & 0 & -\beta & 0 & \beta \\
0 & -\beta & 0 & \beta & 0 \\
-\beta & 0 & \beta & 0 & 1
\end{array}\right) \cdot\left(\begin{array}{l}
c_{0} \\
c_{1} \\
c_{2} \\
c_{3} \\
c_{4}
\end{array}\right)=\left(\begin{array}{l}
0 \\
0 \\
0
\end{array}\right)
$$

where $\beta=\frac{1}{5}-\lambda \in\left(0, \frac{1}{5}\right)$. As before, we guess a solution:

$$
h(x, y)=\left(x^{2}+(2+u) x y+y^{2}\right)\left(x^{2}+(2+v) x y+y^{2}\right)
$$

with $u \neq v>0$, and note that (4.6) is satisfied if

$$
-1-\beta+\beta(6+2 u+2 v+u v)=0 .
$$


It can be verified that the choice $u=\frac{1-5 \beta}{3 \beta}$ and $v=\frac{1-5 \beta}{1+\beta}$ meets this criterion.

\section{Conjectures And OPEN QUestions}

We believe that unique signatures do not exist in $F_{2,2 s}$ when $s \geq 4$. Is there an analogue for Theorem 4.2 in $F_{2,2 s}$ when $s \geq 3$ ? (Even for sextics, this seems to be difficult.) Are there forms with signature $(s, s)$ which do not split into a product of real forms? Is the existence of multiple signatures always a singular phenomenon, or might they, for example, occur in a neighborhood? How can one characterize forms $p$ for which $S(p)=S\left(H_{p}\right)$ ? Do there exist forms with more than two signatures or with signatures $(a, b),(c, d)$ for which $a+b \neq c+d$ ? (They would have to occur in degree $\geq 8$.) What jumps are possible for jump signature sequences? What happens in $F_{3,4}$, the other case in which catalecticants play a role in determing membership in $Q_{n, 2 s}$ ? Finally, which properties discussed here are interesting in real closed fields besides $\mathbb{R}$ ?

\section{REFERENCES}

[1] S. Gundelfinger, Zur Theorie der binären Formen, J. Reine Angew. Math., 100 (1886), 413424.

[2] J. P. S. Kung, Gundelfinger's theorem on binary forms, Stud. Appl. Math., 75 (1986), 163169. MR859177 (87m:11020)

[3] J. P. S. Kung and G.-C. Rota, The invariant theory of binary forms, Bull. Amer. Math. Soc. (N.S.), 10 (1984), 27-85. MR722856 (85g:05002)

[4] G. Pólya and G. Szegö, Problems and theorems in analysis. Vol. II, Springer-Verlag, New York, 1976. MR0465631 (57:5529)

[5] V. Powers and B. Reznick, Notes towards a constructive proof of Hilbert's theorem on ternary quartics, Quadratic forms and their applications, Dublin, 1999 (A. Ranicki, ed.), Contemp. Math., vol. 272, Amer. Math. Soc., Providence, RI, 2000, pp. 209-227. MR 1803369 (2001h:11049)

[6] B. Reznick, Sums of even powers of real linear forms, Mem. Amer. Math. Soc. 96, no. 463 (1992). MR 1096187 (93h:11043)

[7] B. Reznick, Homogeneous polynomial solutions to constant coefficient PDE's, Adv. Math., 117 (1996), 179-192. MR1371648 (97a:12006)

[8] B. Reznick, The length of binary forms, in preparation.

[9] J.J. Sylvester, An Essay on Canonical Forms, Supplement to a Sketch of a Memoir on Elimination, Transformation and Canonical Forms, originally published by George Bell, Fleet Street, London, 1851; Paper 34 in Mathematical Papers, Vol. 1, Chelsea, New York, 1973. Originally published by Cambridge University Press in 1904.

[10] J. J. Sylvester, On a remarkable discovery in the theory of canonical forms and of hyperdeterminants, originally in Philosophical Magazine, vol. 2, 1851; Paper 42 in Mathematical Papers, Vol. 1, Chelsea, New York, 1973. Originally published by Cambridge University Press in 1904 .

[11] J. J. Sylvester, On an elementary proof and generalization of Sir Isaac Newton's hitherto undemonstrated rule for the discovery of imaginary roots, Proc. Lond. Math. Soc. 1 (1865/1866), 1-16; Paper 84 in Mathematical Papers, Vol. 2, Chelsea, New York, 1973. Originally published by Cambridge University Press in 1908.

Department of Mathematics, University of Illinois at Urbana-Champaign, Urbana, ILLINOIS 61801

E-mail address: reznick@math.uiuc.edu 